\title{
Midazolam and ropivacaine act synergistically to inhibit bone cancer pain with different mechanisms in rats
}

\author{
CHI-HUA GUO ${ }^{1 *}$, LU BAI ${ }^{2 *}$, HUANG-HUI WU ${ }^{3 *}$, JING YANG $^{4}$, GUO-HONG CAI ${ }^{5}$, \\ SI-XIANG ZENG ${ }^{1}$, XIN WANG $^{5}$, SHENG-XI WU ${ }^{5}$ and WEI MA ${ }^{1}$ \\ Departments of ${ }^{1}$ Orthopedics and ${ }^{2}$ Medical Imaging, The First Affiliated Hospital \\ of Xi'an Jiaotong University, Xi'an, Shaanxi 710061; ${ }^{3}$ Department of Anesthesiology, \\ Fuzhou General Hospital of Nanjing Military Region, Fuzhou, Fujian 350025; \\ ${ }^{4}$ Institute of Neuroscience, School of Medicine, Zhejiang University, Hangzhou, Zhejiang 310058; \\ ${ }^{5}$ Department of Neurobiology and Collaborative Innovation Center for Brain Science, \\ Fourth Military Medical University, Xi'an, Shaanxi 710032, P.R. China
}

Received May 27, 2016; Accepted October 24, 2016

DOI: 10.3892/or.2016.5241

\begin{abstract}
Analgesic strategy of a single drug analgesia in bone cancer pain (BCP) has shifted to combined analgesia with different drugs which have different mechanism. After tumor cell inculation, the activation of signal transducer and activator of transcription (STAT3) and extracellular signalregulated kinase (ERK) signaling pathway are involved in the development and maintenance of $\mathrm{BCP}$, whereas a decrease in the expression of spinal STAT3 and ERK through using their specific blocker, lead to attenuation of BCP. Hence, in this study, we clarified that intrathecal (i.t.) injection of midazolam (MZL) and ropivacaine (Ropi) induces synergistic analgesia on BCP and is accompanied with different mechanisms of these analgesic effect. Hargreaves heat test was used to detect the analgesic effect of single dose of i.t. MZL, Ropi and their combination on the BCP rats. At consecutive daily administration experiment, thermal hyperalgesia was recorded, and
\end{abstract}

Correspondence to: Professor Sheng-Xi Wu, Department of Neurobiology and Collaborative Innovation Center for Brain Science, Fourth Military Medical University, Xi'an, Shaanxi 710032, P.R. China E-mail: 821496575@qq.com

Professor Wei Ma, Department of Orthopedics, The First Affiliated Hospital of Xi'an Jiaotong University, 277 West Yanta Road, Xi'an, Shaanxi 710061, P.R. China

E-mail: moon007qq@163.com

Abbreviations: BCP, bone cancer pain; MZL, midazolam; Ropi, ropivacaine; $\mathrm{SDH}$, spinal dorsal horn; GFAP, glial fibrillary acidic protein; IBA-1, ionized calcium binding adapter molecule-1; ERK, extracellular signal-regulated kinase; STAT3, signal transducer and activator of transcription

Key words: midazolam, ropivacaine, analgesic, neuron-neuroglia activation, bone cancer pain, ERK and STAT3 pathway immunohistochemical staining was used to detect the expression of c-Fos, spinal glial fibrillary acidic protein (GFAP) and ionized calcium binding adapter molecule-1 (IBA-1). Then, western blot analysis was used to examine spinal TSPO, GFAP, IBA-1, pERK/ERK and pSTAT3/STAT3 levels on day 14 after tumor cell inoculation. i.t. MZL or Ropi showed a short-term analgesia dose-dependently, and MZL displayed better effect on inhibition of pSTAT3 expression than pERK, but Ropi was just the reverse, then consecutive daily administrations of their combination acted synergistically to attenuate thermal hyperalgesia with downregulated spinal 'neuron-astrocytic activation' in the BCP rats. i.t. co-delivery of MZL and Ropi shows synergistic analgesia on the BCP with the inhibition of spinal 'neuron-astrocytic activation'. Spinal different signaling pathway inhibition for MZL and Ropi may be involved in this process.

\section{Introduction}

Bone cancer pain (BCP) is a major clinical intractable problem, seriously affecting the lives of the patients (1). Despite increasing development of basic and clinical research for BCP, the novel clinical analgesics and advanced analgesic strategies often provide inadequate analgesia effect with unacceptable side-effects (2). Thus, in-depth studies are urgently needed for finding more effective and rational treatment strategy for cancer pain.

Recent basic studies in chronic neuropathic and inflammatory pain models suggests that peripheral nerve injury or local inflammatory stimulus results in the pathology changes of the spinal ERK and STAT3, whereas, the inhibition of both ERK and STAT3 contribute to attenuate hyperpathia (3-6). Midazolam (MZL), a short-acting and water-soluble benzodiazepine, is used as anesthetic for sedation, antianxiety, hypnosis of premedication, and induction and maintenance of general anesthesia (7). MZL is known as an adjuvant that has many advantages in clinical practice, such as quick effect and rapid inactivation (8). However, the unacceptable neurotoxicity 
have been found at high doses of MZL, many studies confirm that i.t. MZL produce apoptosis of neurons, and these results restrict the wide use of MZL (9).

Ropivacaine (Ropi), a safe amino amide local anesthetic (LA), is widely used in local anesthesia and postoperative pain control, because it has less impact on motor function and fewer acceptable side-effects in a low concentration (10). However, repeated i.t. Ropi induces catastrophic neurotoxicity and neuronal apoptosis in a dose-dependent manner $(11,12)$. Hence, how to amplify its analgesic effect and reduce the unacceptable side-effects becomes a new research area. Thus, the combination of two different drugs with different and/or overlapped mechanism was considered an effective and harmless method for analgesia in the clinic.

However, it is not clear that the synergistic analgesia of MZL and Ropi on BCP rats and those related underlying mechanisms. Based on the following evidence, we suggested thay combination of MZL\&Ropi might induce synergistic analgesia. First, Ropi as a block of fast voltage-gated sodium channels on neuronal axons is used as analgesia for many kinds of pain in basic experiments $(13,14)$ and clinical trials $(15,16)$. In our previous study, Ropi c alleviated CFA-induced chronic inflammatory pain in rats, and combination with Ropi and Dex showed synergistic analgesia on the chronic inflammatory pain (14). Second, the increased expression of peripheral-type benzodiazepine receptors (PBRs, also named translocator protein, TSPO) are detected in astrocytes and microglia in inflammatory or nerve injury models $(17,18)$. Ro5-4864, an agonist ligand of TSPO can attenuate pain behavior in neuropathic pain, and MZL is also considered as a ligand for TSPO $(19,20)$. Besides, analgesic effect of MZL has been confirmed by previous study in neuropathic pain $(21,22)$.

Hence, using a model of BCP of female rats, we designed the current experiment to test that i.t. co-delivery of MZL and Ropi at lower dose yields synergistic analgesia, and further explore the underlying analgesic mechanisms of MZL and Ropi. It was found that these two clinically used drugs may be utilized in combination to achieve pain relief in BCP patients.

\section{Materials and methods}

Animal preparation. Adult female Sprague-Dawley (SD) rats, weighing 200-220 g, were provided by Experimental Animal Center of the Xi'an Jiaotong University. All rats were housed in controlled conditions (standard transparent plastic cages with a 12/12-h light/dark cycle, under $22-26^{\circ} \mathrm{C}$ ambient temperature with food and water ad libitum). All rats were allowed to adapt to the housing environment for at least 3 days before the experiments.

All experimental procedures were conducted in accordance with the Animal Use and Care Committee for Research and Education of the Xi'an Jiaotong University and National Institute for Physiological Sciences Animal Care and Use Committee. All efforts were made to minimize suffering of the animals and the number of animals used was carefully controlled.

Cell line and drug administration. Female SD rats were administered intraperitoneal (i.p.) inoculation of Walker
256 mammary gland carcinoma cells $\left(2 \times 10^{6}\right.$ cells $\left./ \mathrm{ml}, 1 \mathrm{ml}\right)$. After 1 week, tumor cells were extracted from the cancerous ascitic fluid of rats, and resuspended in a concentration of $1 \times 10^{7}$ cells $/ \mathrm{ml}$ in $0.01 \mathrm{M}$ phosphate-buffered saline (PBS) for inoculation.

MZL (5 mg/5 ml) and Ropi (100 mg/10 ml) were purchased from Nhwa Pharmaceutical Co., Ltd. (Jiangsu, China) and AstraZeneca AB (Sweden), respectively. These drugs were diluted with artificial cerebral spinal fluid (ACSF: $\mathrm{NaCl}$ $124 \mathrm{mM}$, D-glucose $10 \mathrm{mM}, \mathrm{NaH}_{2} \mathrm{PO}_{4} 1 \mathrm{mM}, \mathrm{NaHCO}_{3}$ $25 \mathrm{mM}, \mathrm{MgSO}_{4} 1 \mathrm{mM}, \mathrm{KCl} 4.4 \mathrm{mM}$ and $\mathrm{CaCl}_{2} \cdot \mathrm{H}_{2} \mathrm{O} 2 \mathrm{mM}$ ) prior to i.t. application. Finally, MZL $(5 \mathrm{mg} / 5 \mathrm{ml})$ was diluted to concentrations $(10 \mu \mathrm{g} / \mathrm{ml})$, and the target concentrations of Ropi $(100 \mathrm{mg} / 10 \mathrm{ml})$ was $1 \mathrm{mg} / \mathrm{ml}$.

BCP model surgery. The inoculation was performed as previously described (23). Briefly, rats were anaesthetized with chloral hydrate $(300 \mathrm{mg} / \mathrm{kg}$, i.p.), the right rear hindlimb was shaved in order to expose the skin over the femoraltibial joint. The intercondylar eminence of the right tibia was exposed after cleaning skin 3 times with iodine tincture and $75 \%$ ethanol. A 22-gauge needle was drilled into the site as described previously, then $20 \mu \mathrm{l}$ microinjection syringe (Hamilton, USA) containing a $10 \mu 1$ suspension of tumor cells was used to inject the tumor cells into the tibial cavity slowly. The drilled hole was sealed with bone wax (Johnson \& Johnson, USA) in order to prevent tumor cells from spreading outside the bone. For the sham group, $10 \mu \mathrm{l}$ PBS replaced tumor cells into the tibia (5).

Intrathecal implantation. Intrathecal implantation was performed under chloral hydrate $(300 \mathrm{mg} / \mathrm{kg}$, i.p.). Briefly, a midline incision $(3 \mathrm{~cm})$ was cut on the back of the rat at the level of the thoracic vertebrae. A pre-measured length of PE-10 tubing was passed caudally from the T8 to the L3 level of the spinal cord, fixed at the back of rat's ears through subcutaneous tunnel, and $2 \mathrm{~cm}$ of the free end was exposed in the upper thoracic region. Rats were allowed to recover for a period of 3-5 days before further use. The animals judged as with no neurological deficit and that presented complete paralysis of the tail and bilateral hind legs after administration of $2 \%$ lidocaine $(10 \mu \mathrm{l})$ through the intrathecal catheter were used for the following experiments.

Behavioral test. Thermal hyperalgesia was evaluated by Hargreaves test. In brief, rats were placed individually in crylic boxes with wire mesh floors and allowed to adapt for $30 \mathrm{~min}$. The radiant heat stimulator was used to stimulate the plantar surface of the hindlimb paw, and the intensity of the beam was set to produce a basal paw withdrawal latency (PWL) of approximate $16 \mathrm{sec}$. The PWLs were recorded according to previous studies above. To prevent tissue thermal burn, the cut-off value was set at $20 \mathrm{sec}$. The blinding method was implemented strictly for the whole behavioral test.

Motor coordination was determined using a standard rat rotarod test (Shanghai Mobiledatum Information Technology Co., Ltd., China). Daily training for each rat was performed at $30 \mathrm{~min}$ after intrathecal administration of compound for 7 days, and measured as following methods: rats were placed on the rotating drums and the time was measured from the 
start of the acceleration period until the rat fell off the drum. A cut-off latency was $30 \mathrm{sec}$ in all rotarod assessments. The time that the animal remained on the rotarod was recorded and expressed as a percentage of its own baseline value.

Immunofluorescence histochemistry staining. Rats were deeply anesthetised with overdose chloral hydrate $(10 \%$, $0.3 \mathrm{ml} / 100 \mathrm{~g}$ ) and transcardially perfused with $200 \mathrm{ml}$ of $0.01 \mathrm{M}$ PBS (pH 7.4), followed by $500 \mathrm{ml}$ of $4 \%$ paraformaldehyde in $0.1 \mathrm{M}$ phosphate buffer (PB, pH 7.4). The L4-L6 spinal cord segments were harvested and dehydrated in $30 \%$ sucrose at $4^{\circ} \mathrm{C}$. Transverse spinal sections (30 $\mu \mathrm{m}$ thickness) were then cut on a cryostat (CM3050S, Leica, Germany). For double immunofluorescence, the sections were incubated with mouse anti-c-Fos (1:500, Abcam, USA), mouse anti-GFAP (1:500, Millipore, USA) and goat anti-IBA-1 (1:500, Abcam), followed by FITC-conjugated secondary antibodies (1:500, Invitrogen, USA) for $3 \mathrm{~h}$ at room temperature. The stained sections were observed and captured with a confocal laser canning microscope (FV1000, Olympus, Japan).

Western blot analysis. The L4-L6 spinal cord segments were rapidly extracted from anesthetized rats. The tissues were homogenized in lysis buffer (Bio-Rad Laboratories, USA) which contain a mixture of protease inhibitors and phenylmethylsulfonyl fluoride (Roche Diagnostics, Switzerland). Equivalent amounts of protein $(10 \mu \mathrm{l})$ were loaded and separated by $10 \%$ sodium dodecyl sulfate-polyacrylamide gel electrophoresis (10\% SDS-PAGE; Bio-Rad), transferred to PVDF membranes (Millipore). The membrane was incubated with primary antibody overnight at $4^{\circ} \mathrm{C}$, followed by HRP-conjugated secondary antibodies for $2 \mathrm{~h}$ $(1: 5,000$, CST $)$. The secondary antibody signal was detected with enhanced chemiluminescence (Advansta, USA), and captured by Omega Lum G system (Aplegen, USA). The following primary antibodies were used in our studies: goat anti-TSPO (1:1,000, Sigma-Aldrich, USA), rabbit antipERK (1:1,000, Abcam), mouse anti-ERK (1:1,000, Abcam), rabbit anti-pSTAT3 (1:2,000, CST, USA), rabbit anti-STAT3 (1:2,000, CST, USA), mouse anti-GFAP (1:1,000, Millipore), goat anti-IBA-1 (1:1,000, Abcam) and mouse anti- $\beta$-actin (1:10,000, Sigma-Aldrich).

Statistical analysis. All data were expressed as mean \pm standard error mean (SEM), and analyzed by researchers with double-blind method. One-way analysis of variance (ANOVA) with Dunnett's multiple comparison post hoc test was used for multiple comparison between groups for area under the curve. Two-way ANOVA with Bonferroni post hoc test was used for measures of dose by time and rotarod. Variable slope nonlinear regression was used for dose-response curves by using Graph-Pad Prism 6.0 (Graph-Pad software, San Diego, CA, USA). Isobolographic analysis was based on Tallarida and our previous study (24). $\mathrm{P}<0.05$ was considered as statistically significant.

\section{Results}

Effect of i.t. MZL on BCP. In order to identify the potential analgesic effect of MZL in BCP, different doses of MZL were i.t. injected on day 14 after tumor cell inoculation. Based on previous studies, MZL was given in doses of 10, 20, 40 and $80 \mathrm{ng}$ and PWLs were measured at 30, 60, 90, 120, 150 and 180 min post-injection of MZL and vehicle. Compared with BCP-Veh group, i.t. MZL significantly elevated PWLs in a dose-dependent manner with a peak effect at dose of $80 \mathrm{ng} / \mathrm{kg}$ at 60 min post-injection (Fig. $1 \mathrm{~A}$ and $\mathrm{B} ; \mathrm{P}<0.01$ ). The $\mathrm{ED}_{50}$ for MZL was calculated as $22.6 \mathrm{ng} / \mathrm{kg}$ based on the log (dose) vs. response curve (Fig. $1 \mathrm{C}$ and $\mathrm{D} ; \mathrm{P}<0.01$ ). Besides, the rotarod test revealed $22.6 \mathrm{ng} / \mathrm{kg}$ MZL did not affect the motor performance of rats compared to vehicle treatment at any of the time-points tested (Fig. 2E; P<0.01). Hence, i.t. MZL inhibited thermal hyperalgesia in $\mathrm{BCP}$ rats.

Effect of i.t. Ropi on BCP. According to our previous studies (14), increasing doses of Ropi were given in 12.5, 25, 50 and $100 \mu \mathrm{g} / \mathrm{kg}$ and PWLs were recorded at 30, 60, 90, 120, 150 and 180 min post-injection of Ropi and vehicle. Compared with BCP-Veh group, i.t. Ropi also significantly elevated PWLs in a dose-dependent manner with a peak effect at dose of $100 \mu \mathrm{g} / \mathrm{kg}$ at $30 \mathrm{~min}$ post-injection (Fig. 1E and $\mathrm{F} ; \mathrm{P}<0.01)$. The $\mathrm{ED}_{50}$ for Ropi was calculated as $43.3 \mu \mathrm{g} /$ $\mathrm{kg}$ based on the $\log$ (dose) vs. response curve (Fig. 1G and $\mathrm{H} ; \mathrm{P}<0.01)$. Similarly, the rotarod test also revealed $43.3 \mu \mathrm{g} / \mathrm{kg}$ Ropi did not affect the motor performance of rats compared to vehicle treatment at any of the time-points tested (Fig. 2E; P<0.01). Hence, i.t. Ropi apparently inhibits thermal hyperalgesia in a dose-dependent manner in BCP rats.

Effect of i.t. MZL and Ropi combination on BCP. Next, we calculated synergistic analgesic effect of MZL\&Ropi on the basis of the antinociceptive effects showed by two drugs. According to additive regression, we calculated theoretical $\mathrm{ED}_{50}$ for a fixed-ratio (1:1) combination of MZL and Ropi. Hence, the doses of MZL\&Ropi combinations were as follows: 2.26 Dex + 4.33 Ropi (ED 50add*1/10 Dex\&Ropi), 4.52 Dex + 8.66 Ropi ( $\mathrm{ED}_{50 \mathrm{add} * 2 / 10} \mathrm{Dex} \&$ Ropi), $9.04 \mathrm{Dex}+17.32$ Ropi $\left(\mathrm{ED}_{50 \mathrm{add} * 4 / 10}\right.$ Dex\&Ropi) and 18.08 Dex + 34.64 Ropi $\left(\mathrm{ED}_{50 \mathrm{add} * 8 / 10}\right.$ Dex\&Ropi).

PWLs were measured at 30, 60, 90, 120, 150 and $180 \mathrm{~min}$ post-injection of MZL\&Ropi and vehicle. i.t. MZL\&Ropi combination significantly elevated PWLs in a dosedependent manner with a peak effect at dose of 18.08 Dex and 34.64 Ropi at 60 min post-injection (Fig. 2A and B; $\mathrm{P}<0.01$ ). Besides, the averaged valid analgesic duration was significantly dose-dependently prolonged. The experimental $\mathrm{ED}_{50 \mathrm{comb}}$ at this fixed dose ratio calculated from these doseresponse curves for pain responses was 6.61 MZL + 12.67 Ropi (Fig. $2 \mathrm{C}$ and $\mathrm{D}, \mathrm{P}<0.05$ ). Isobolographic analysis showed that MZL\&Ropi acted synergistically to inhibit BCP in rats, because $\mathrm{ED}_{50 \text { comb }}$ was smaller than the lower (95\%) range of $\mathrm{ED}_{50 \text { add }}$ (Fig. 2F, $\mathrm{P}<0.05$ ). These results revealed that the combination of MZ\&Ropi facilitated to prolong analgesic duration and enhance analgesic intensity with less dose of MZL and Ropi. The combination of MZL\&Ropi did not affect motor coordination of the animals (Fig. 2E; P<0.01).

Continuous analgesia of i.t. MZL\&Ropi on BCP. Above the effect of i.t. MZL, and Ropi, or their combination was described 
A

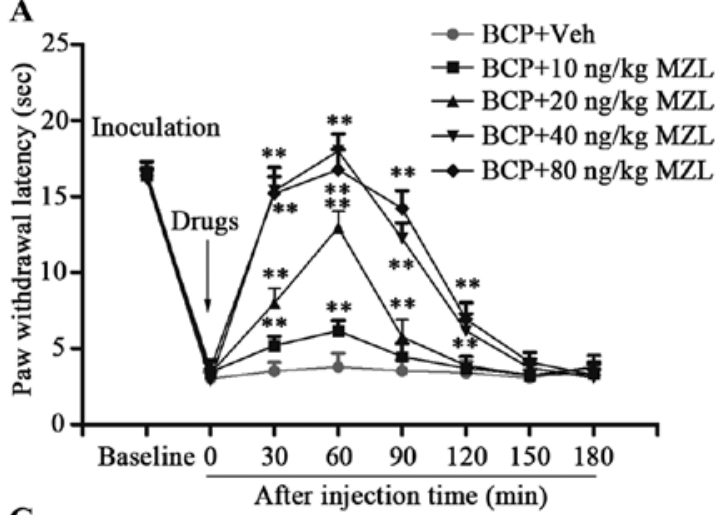

C

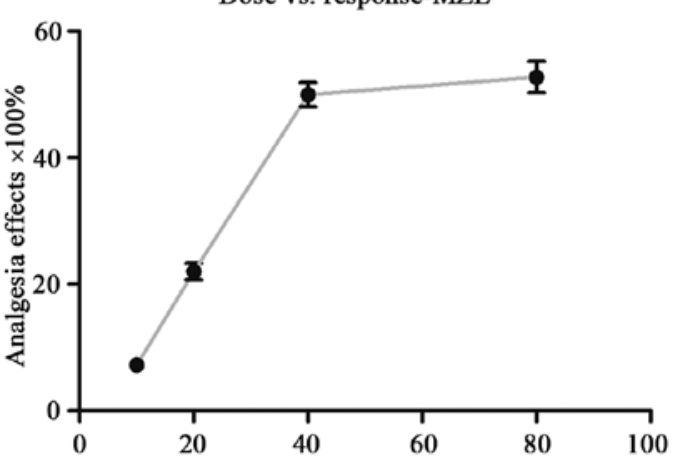

E $\quad$ Midazolam $(\mathrm{ng} / \mathrm{kg})$

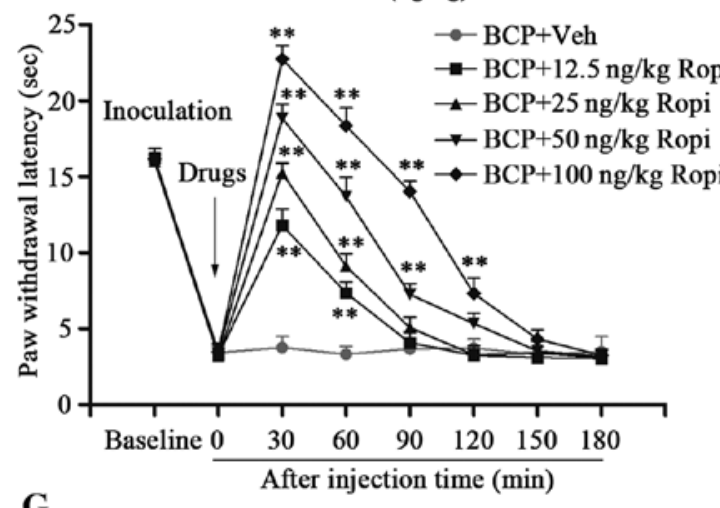

G

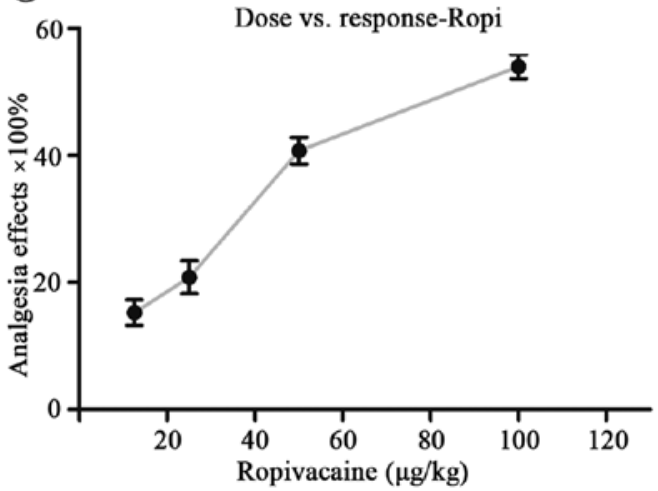

B

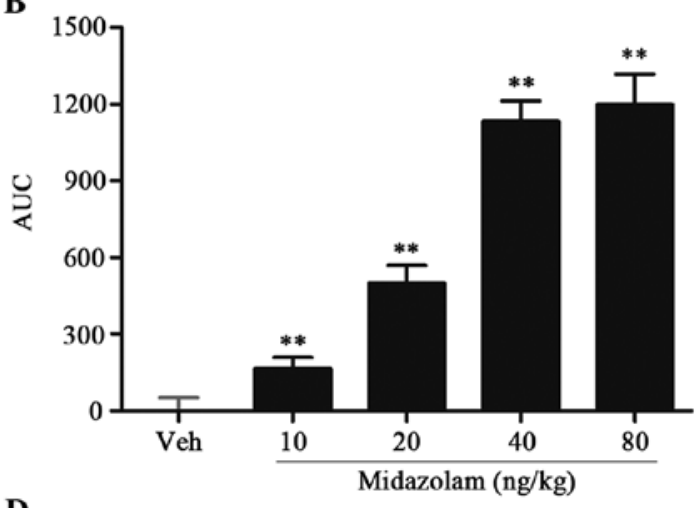

D

Log (dose) vs. response-MZL

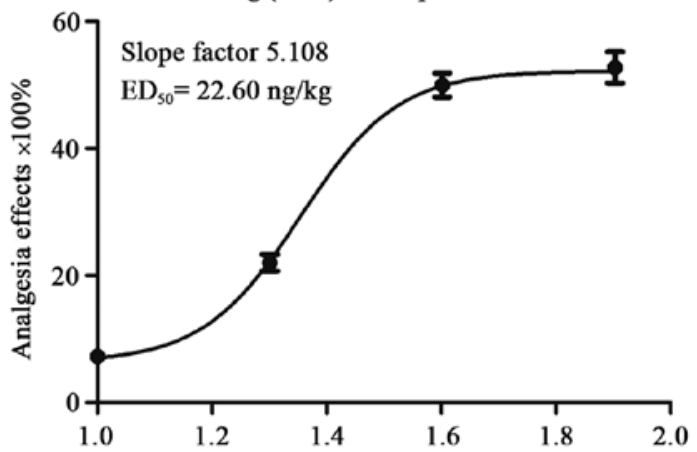

F Log (midazolam)

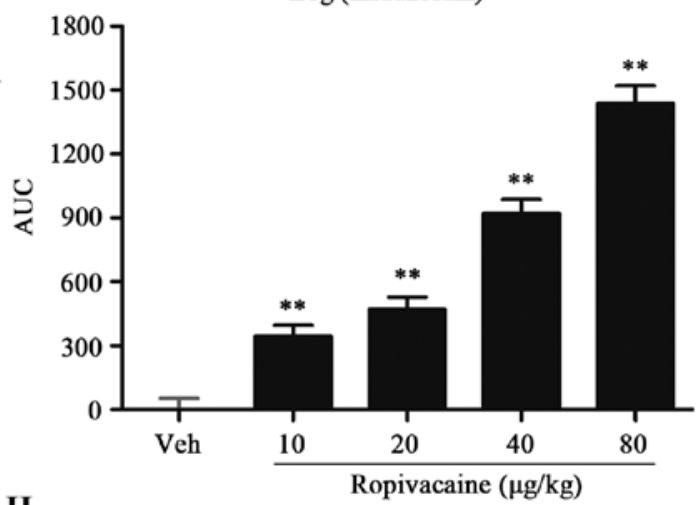

H

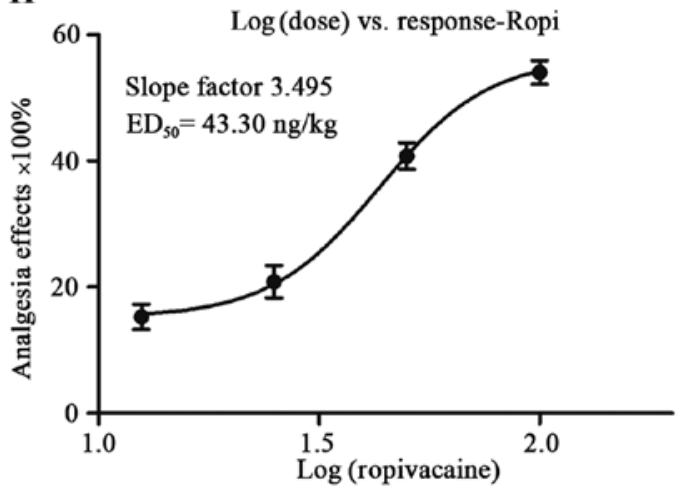

Figure 1. i.t. MZL or Ropi dose-dependently inhibited thermal hyperalgesia in bone cancer pain. Analgesia effects of different doses of i.t. MZL or Ropi was observed (A and E). The AUCs of i.t. MZL or Ropi were calculated to perform statistical analysis (B and F). MZL or Ropi dose-dependently inhibited thermal hyperalgesia with the dose-effect $(\mathrm{C})$ or $(\mathrm{G})$ and $\mathrm{EC}_{50}(\mathrm{D})$ or $(\mathrm{H})$, respectively. ${ }^{*} \mathrm{P}<0.05 ;{ }^{* *} \mathrm{P}<0.01$. Compared with $\mathrm{BCP}-\mathrm{Veh}$ group.

in a short-term observation. Then, their analgesia on $\mathrm{BCP}$ induced chronic hyperalgesia was further explored. According to $\mathrm{ED}_{50} \mathrm{MZL}, \mathrm{ED}_{50}$ Ropi, and $\mathrm{ED}_{50 \text { comb }}$ Dex\&Ropi, the dose regimens were as follows: BCP-MZL group, $22.6 \mathrm{ng} / \mathrm{kg}$ MZL; BCP-Ropi group, $43.3 \mu \mathrm{g} / \mathrm{kg}$ Ropi; and BCP-MZL\&Ropi group, $6.61 \mathrm{ng} / \mathrm{kg}$ MZL and $12.67 \mu \mathrm{g} / \mathrm{kg}$ Ropi. 
A

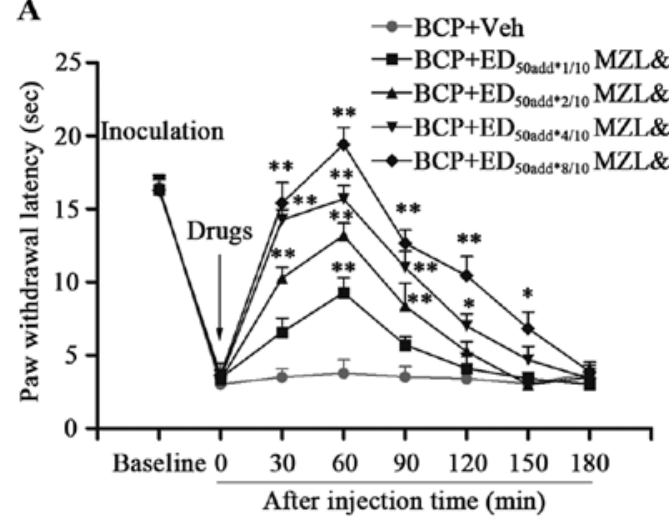

C

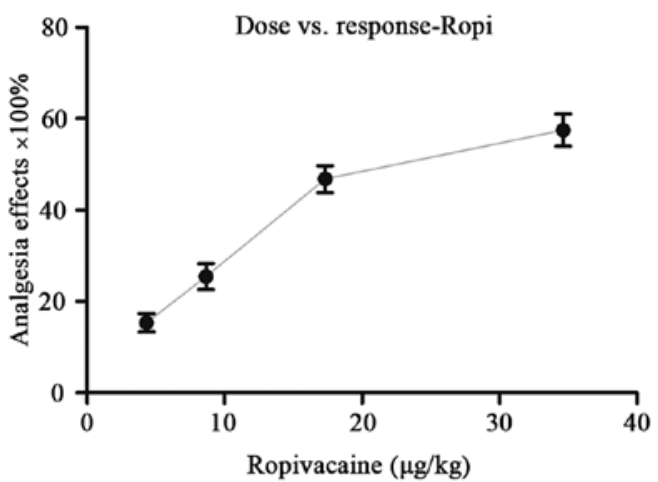

E

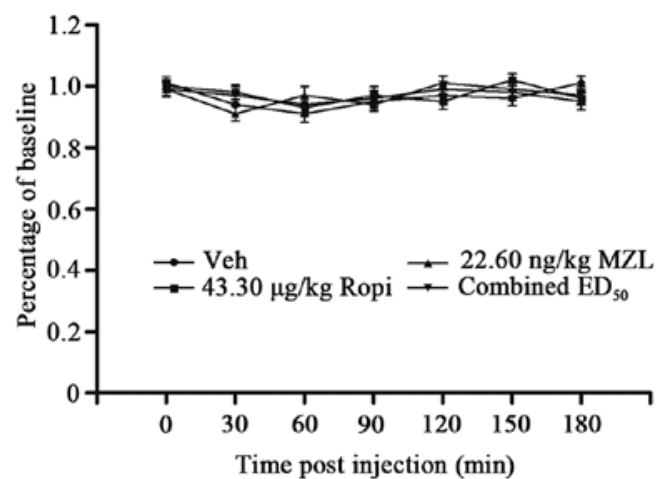

G

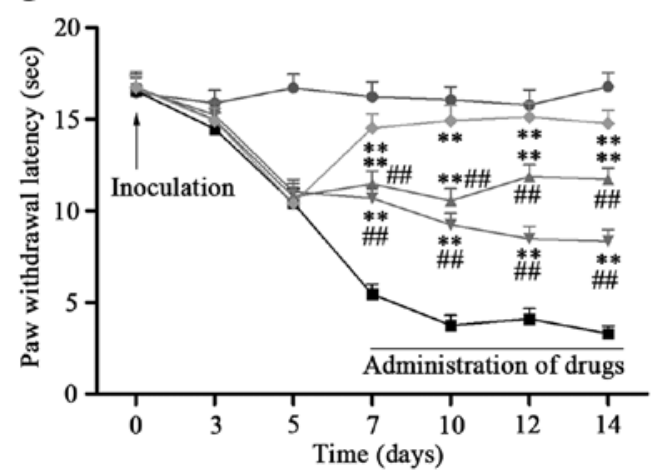

B

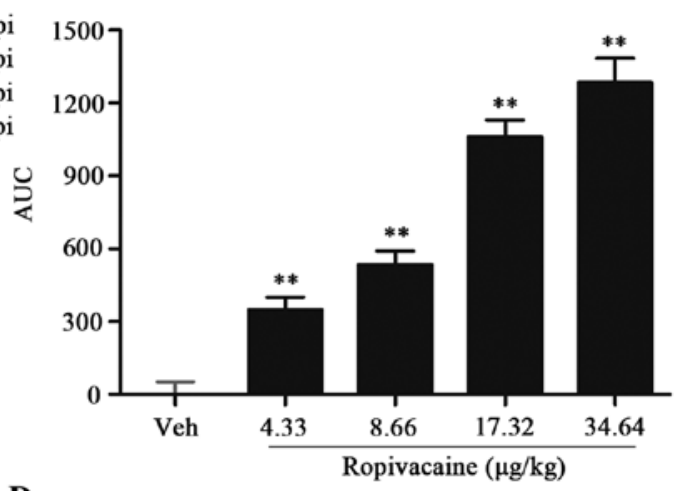

D

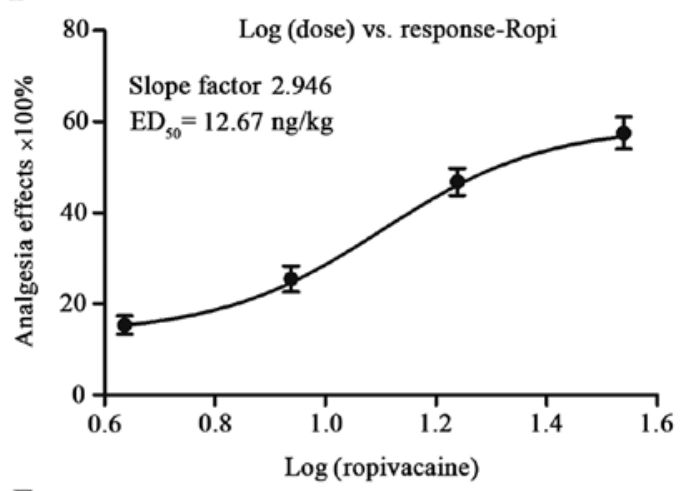

F

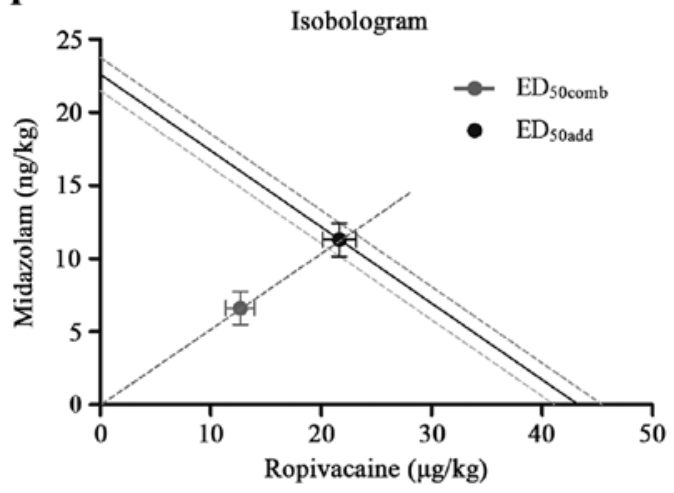

$\rightarrow$ Sham

$\rightarrow$ BCP+Veh

$\leadsto$ BCP+Ropi

$\rightarrow \mathrm{BCP}+\mathrm{MZL}$

$\rightarrow$ BCP+MZL\&Ropi

Figure 2. i.t. MZL and Ropi combinations dose-dependently inhibit thermal hyperalgesia in bone cancer pain. Analgesia effects of different doses of i.t. MZL and Ropi combinations are shown (A). The AUC of i.t. MZL and Ropi combinations was observed (B). MZL and Ropi combinations dose-dependently inhibited thermal hyperalgesia with the dose-effect $(C)$ and $\mathrm{EC}_{50}(\mathrm{D})$. The effects of i.t. MZL and Ropi combinations on motor performance in normal rats $(\mathrm{E})$. Iosobologram for combination analgesia is presented (F). Long-term analgesia effect of i.t. MZL, Ropi, or their combination on bone cancer pain during 8 days after tumor cell inoculation, respectively $(\mathrm{G}) .{ }^{*} \mathrm{P}<0.05 ;{ }^{* *} \mathrm{P}<0.01$. Compared with BCP-Veh group. ${ }^{\# \#} \mathrm{P}<0.01$. Compared with BCP-MZL\&Ropi group.

A significant analgesic effect of all intervention groups was observed. Compared with BCP-Vehicle group, i.t. MZL, Ropi, or their combination significantly increased the PWLs. In these three group, BCP-MZL\&Ropi groups performed better analgesic effect than the others, however, analgesic effect of BCP-MZL group gradually attenuated with time. These results 
A

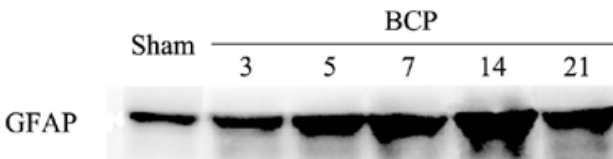

IBA-

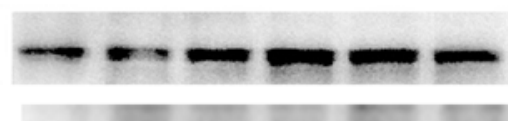

TSPO

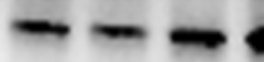

$\beta$-actin

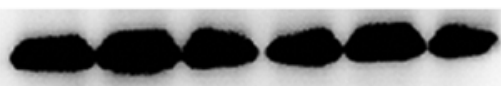

pERK

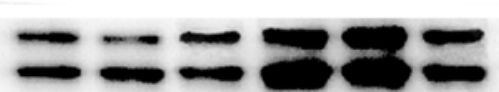

ERK
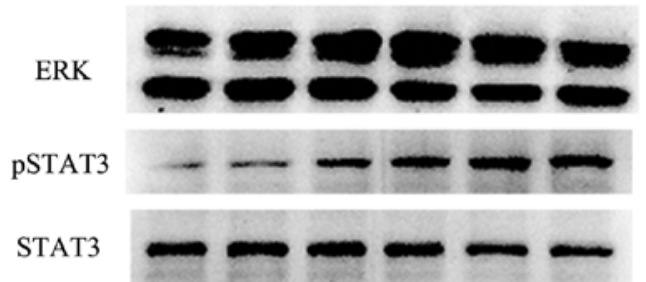

B
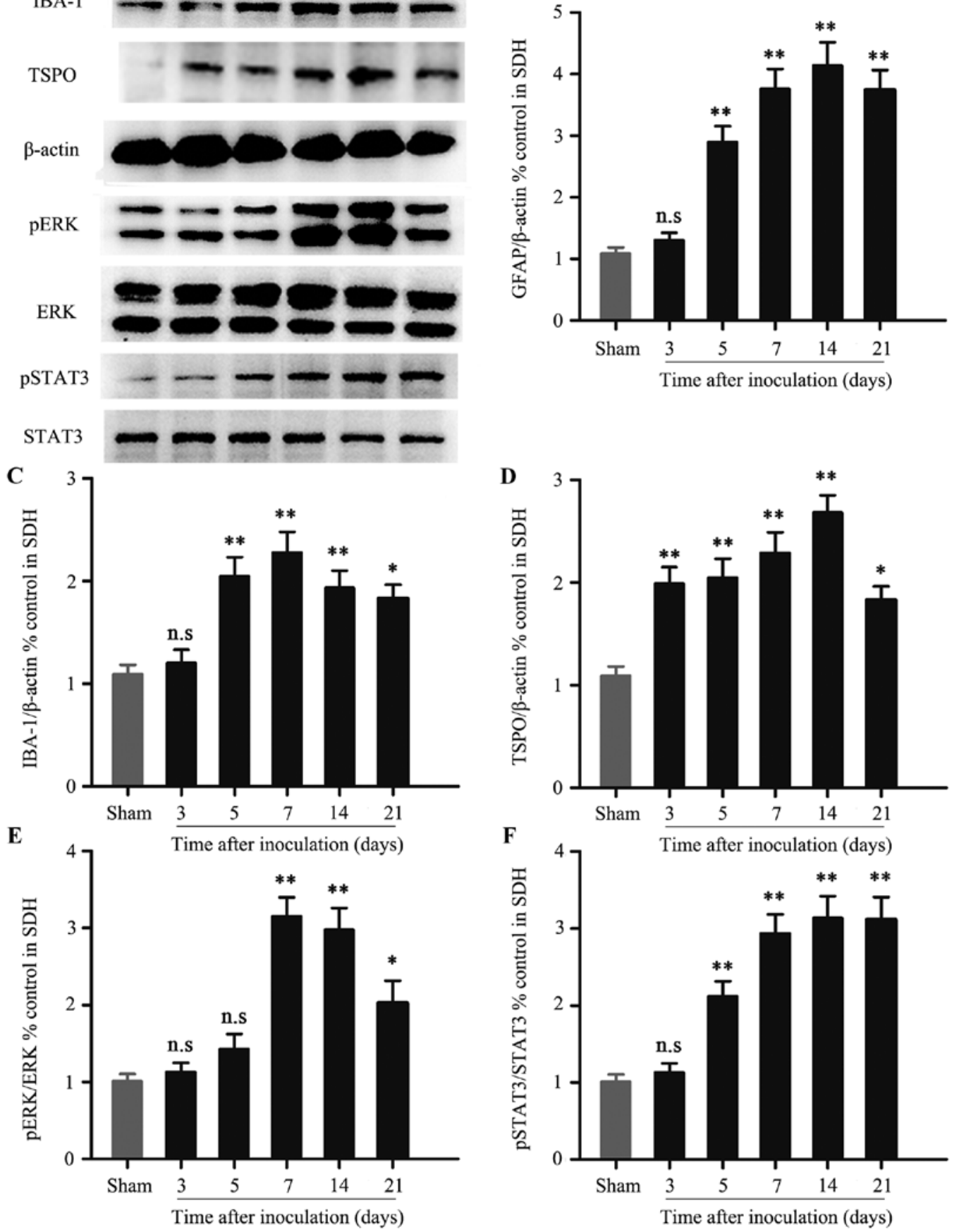

Figure 3. Expression of spinal GFAP, IBA-1, TSPO, pERK and pSTAT3 in BCP rats. Western blot analysis of GFAP, IBA-1, TSPO, pERK and pSTAT3 expression on days 3, 5, 7, 14 and 21 after tumor cells inoculation is shown (A). Results of western blot analysis are presented (B-F). ${ }^{*} \mathrm{P}<0.05$; ${ }^{* * *} \mathrm{P}<0.01$. Compared with Sham-Veh group.

suggested that i.t. MZL\&Ropi combination showed better analgesic effect in a long-term treatment in $\mathrm{BCP}$ rats with less doses and longer duration than each single drug (Fig. $2 \mathrm{~F}, \mathrm{P}<0.05)$.

Expression of spinal TSPO, pERK and pSTAT3 in BCP. We examined the time course of spinal GFAP, IBA-1, TSPO, pERK and pSTAT3 expression by western blot analysis.
Western blot analysis revealed that spinal sustained elevation of GFAP could be detected in chronological order, and was accompanied by the development of behavioral hypersensitivity (Fig. $3 \mathrm{~A}$ and $\mathrm{B} ; \mathrm{P}<0.05$ ). In sharp contrast, the significant peak value of IBA-1 was not observed on day 14, but only on day 7 (Fig. 3A and C; $\mathrm{P}<0.05$ ). Similar to GFAP expression, the expression of TSPO was significantly increased from 

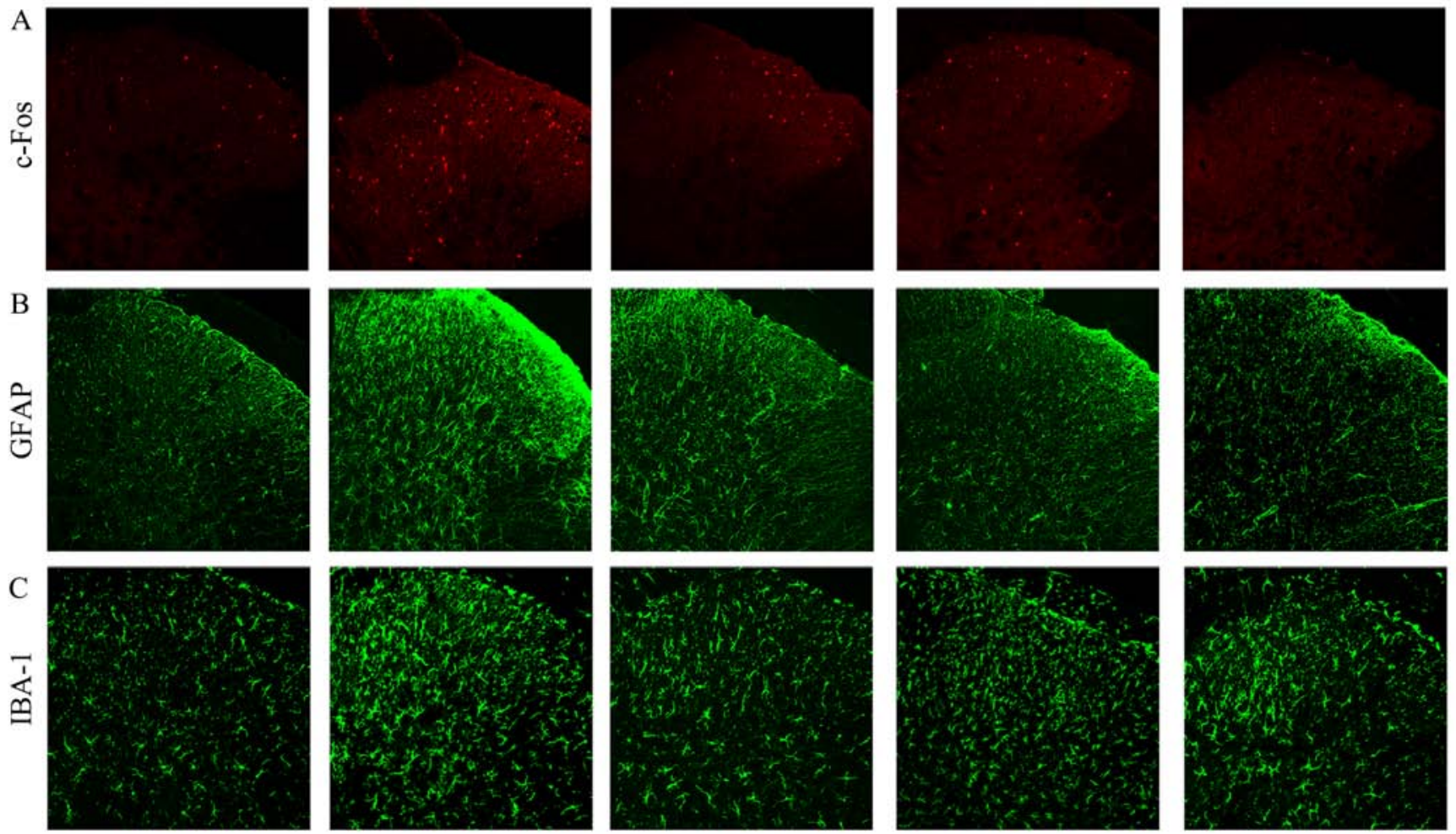

Sham-Veh

BCP-Veh

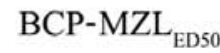

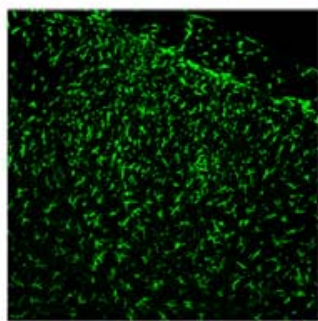

BCP-Ropi ${ }_{\text {ED50 }}$

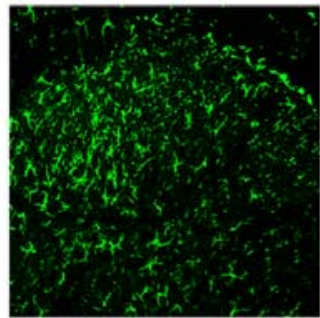

BCP-MZL\&Ropi ${ }_{\text {EDS0 }}$

Figure 4. Effect of i.t. MZL, Ropi or their combination on neuronal and neuroglial activation. Tumor cell injection induced appreciable spinal neuronal (A), astrocytic (B) and microglial (C) activation. Original magnification, $\mathrm{x} 200$.

day 3 to 14 , and reached its peak on day 14 (Fig. 3A and D; $\mathrm{P}<0.05)$. Then, the significant up-regulation of spinal $\mathrm{pERK}$ induced by tumor cell inculation was observed on day 7 (Fig. 3A and E, P<0.05). However, spinal pSTAT3 expression was significantly activated on day 5 compared with the sham group (Fig. 3A and F, $\mathrm{P}<0.05$ ).

Effect of i.t. MZL, Ropi or their combination on neuronal and glial activation. Increasing evidence has confirmed that spinal neuron-astrocytic activation played a vital role in $\mathrm{BCP}$ rat models. Hence, we further explored whether the inhibition of neurons and neuroglia cells contributed to analgesia at doses of $\mathrm{ED}_{50} \mathrm{MZL}, \mathrm{ED}_{50}$ Ropi, or $\mathrm{ED}_{50 \mathrm{comb}}$ MZL\&Ropi which achieved equal $50 \%$ analgesia.

We administered i.t. $\mathrm{ED}_{50} \mathrm{MZL}, \mathrm{ED}_{50}$ Ropi, or $\mathrm{ED}_{50 \mathrm{comb}}$ MZL\&Ropi once daily from day 7 to 14 to observe the effects on astrocytic and microglia activation in BCP rats on day 14. Western blot analysis confirmed that the increased GFAP expression could be inhibited by each intervention group compared with BCP group. However, the GFAP expression for BCP-MZL\&Ropi group was less than the other two intervention groups, while there was no group difference between BCP-MZL and BCP-Ropi groups (Fig. 5A and B, $\mathrm{P}<0.05)$. Interestingly, the IBA-1 expression for BCP-MZL and BCP-Ropi groups was inhibited by each intervention, but there was no significant difference between BCP and BCP-MZL\&Ropi groups. Compared with BCP-MZL and BCP-Ropi groups, i.t. MZ\&Ropi combine did not inhibit IBA-1 expression (Fig. 5A and $\mathrm{C}, \mathrm{P}<0.05$ ).
In addition, immunohistochemistry showed increased nuclei expression of c-Fos on day 14 in BCP group. In each intervention group, the c-Fos expression was inhibited by two drugs respectively or combination (Fig. 4A). Immunohistochemical expression of GFAP and IBA-1 were similar to western blot results (Fig. 4B and C). These results indicated that a synergistic effect, which was also observed in previous behaviour tests, could be contributed by astrocytes, but not microglia.

Effect of i.t. MZL, Ropi or their combination on $p E R K$ and pSTAT3 expression. Next, we administered i.t. ED $_{50}$ MZL, $\mathrm{ED}_{50}$ Ropi, or $\mathrm{ED}_{50 \mathrm{comb}}$ MZL\&Ropi once daily from day 7 to 14 to observe the effects on pERK and pSTAT3 activation in SDH on day 14. Analysis of the pERK expression inhibited by each intervention group was compared with BCP group. However, the pERK expression for BCP-Ropi and BCP-MZL\&Ropi groups were less than BCP-MZL groups, and there was no group difference between BCP-Ropi and BCP-MZL\&Ropi groups (Fig. 5A and $\mathrm{D}, \mathrm{P}<0.05$ ). The pSTAT3 expression inhibited only by BCP-MZL group compared with BCP group, but there was great significant difference between BCP-Ropi and BCP-MZL\&Ropi groups. The inhibition of pSTAT3 was the strongest in BCP-MZL group, compared with BCP-Ropi and BCP-MZL\&Ropi group (Fig. 5A and E, P<0.05). These results indicated that i.t. MZL could inhibit pSTAT3 expression much better than i.t. Ropi, and i.t. Ropi was just the opposite compared with MZL, i.t. Ropi showed better inhibition of pERK than i.t. MZL. Based on previous behavior tests on intervention groups, we suggest that analgesia effect of MZL 
A

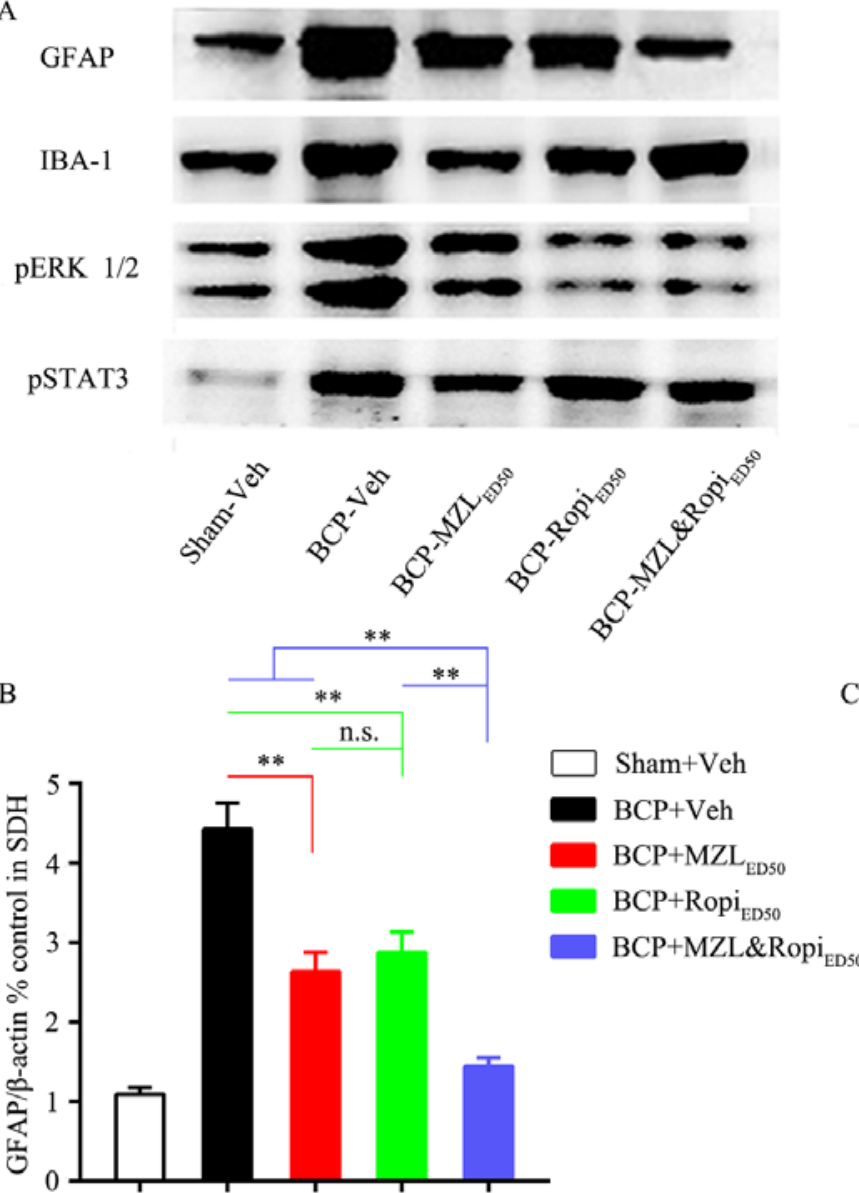

$\mathrm{D}$

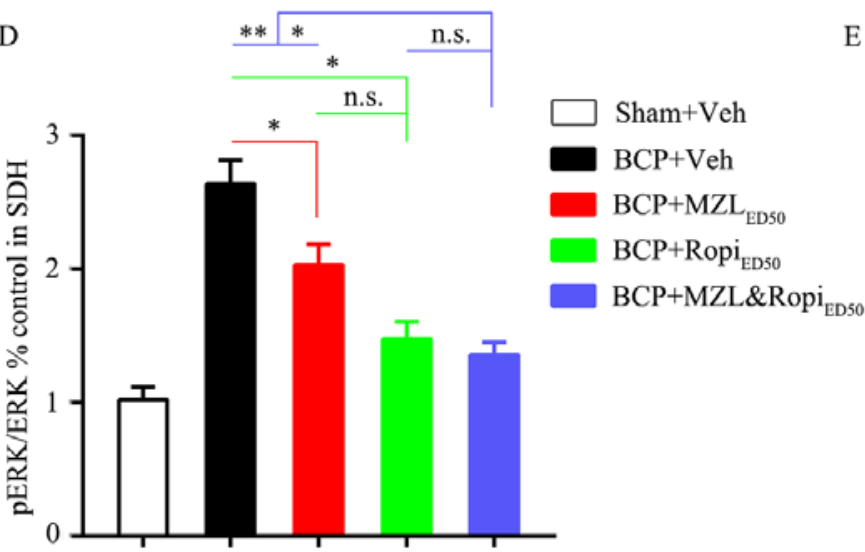

$\mathrm{C}$
STAT3

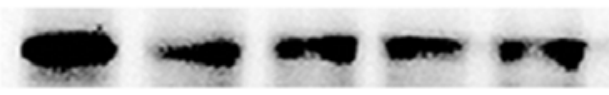

ERK $1 / 2$

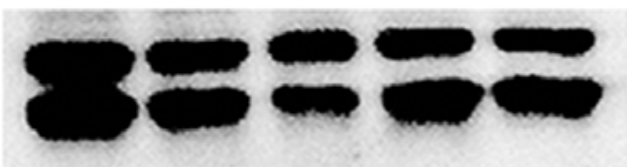

$\beta$-actin
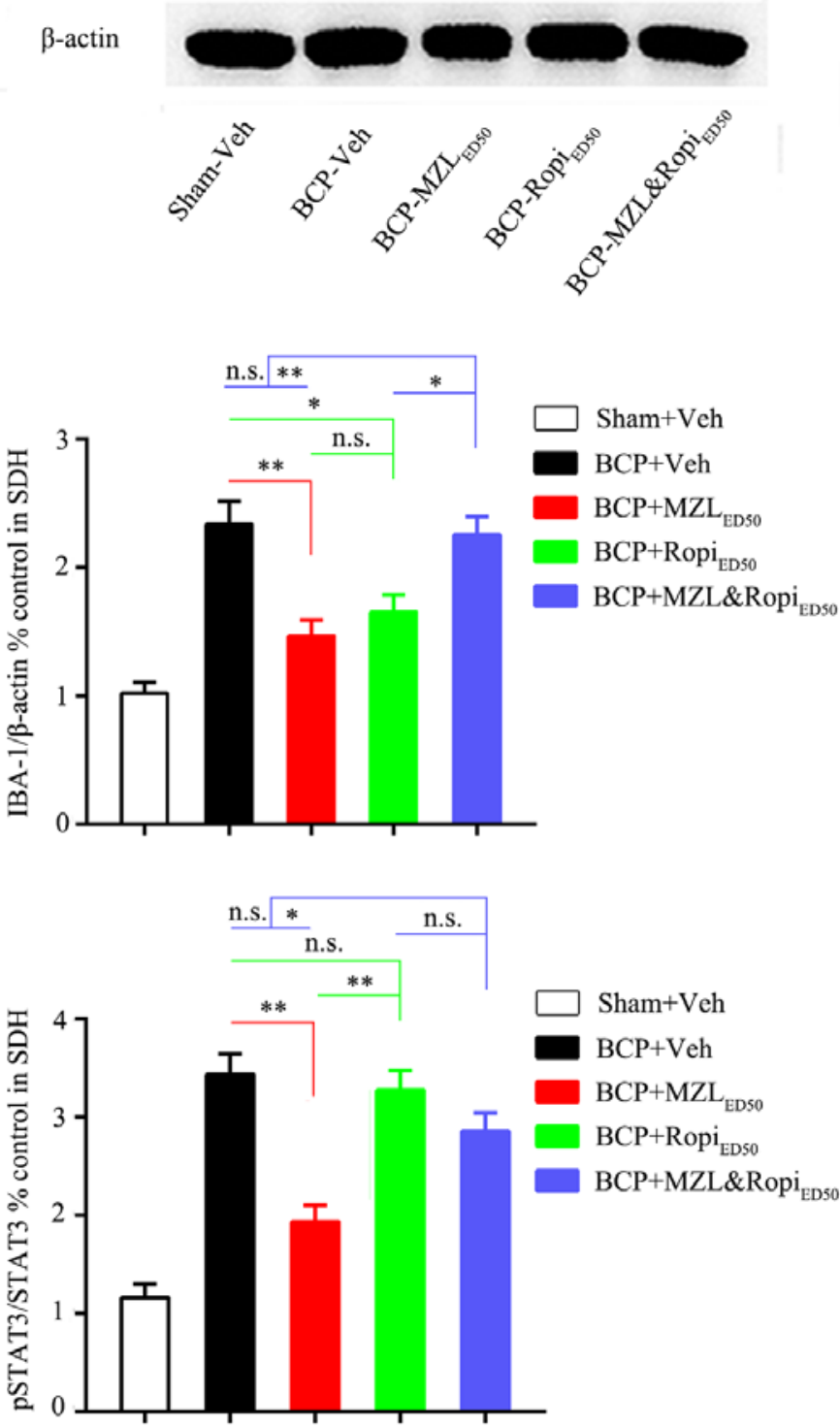

Figure 5. Effect of i.t. Dex, Ropi, or their combination on spinal GFAP, IBA-1, pERK and pSTAT3 in BCP rats. Western blot analysis of GFAP, IBA-1, pERK and pSTAT3 expression on day 14 after tumor cell inoculation is shown (A). Results of western blot analysis are presented (B-E). ${ }^{*} \mathrm{P}<0.05$; $^{* *} \mathrm{P}<0.01$.

could be contributed to inhibition of pSTAT3, and analgesic effect of Ropi was relevant to inhibition of pERK, furthermore, analgesic effect of MZL and Ropi combination could result from pERK inhibition, because it had stronger inhibitory effect on pERK expression compared with pSTAT3.

\section{Discussion}

Our study showed that MZL and Ropi reduced thermal hyperalgesia in a dose-dependent manner in BCP rats, respectively.
Even more importantly, the low dose combination of MZL and Ropi acted synergistically to reduce thermal hyperalgesia after tumor cell inoculation. Next, we found that spinal neuron-astrocytic activation played a vital role in BCP rats. MZL dominantly downregulated the level of pSTAT3 while Ropi inhibited the spinal pERK expression. Therefore, the combined use of MZL and Ropi might be a new therapeutic approach for treatment of BCP.

Recent studies report that benzodiazepine receptor consists of two types of receptors, one is central-type benzo- 
diazepine receptor (CBRs), which are coupled to type A $\gamma$-amminobutyric acid $\left(\mathrm{GABA}_{\mathrm{A}}\right)$ receptor, and the other is peripheral-type benzodiazepine receptors (PBRs, also named translocator protein, TSPO), and these two types of receptors are the targets for MZL by increasing GABA release (20). Compared with CBRs that are expressed exclusively in the central nervous system (CNS), low expression of TSPOs in CNS are common in normal condition. However, studies have shown that increased expression of TSPOs in both astrocytes and microglia are generally accompanied by neuroglia activation during the nociceptive stimulation, such as inflammation, CNS injury and pain $(25,26)$. Moreover, Ro5-4864, an agonist of TSPO, but not TSPO antagonist PK11195, can attenuate the pain behavior in a dose-dependent manner that is contributed to suppress the astrocytic activation $(17,27,28)$. In this study, similar results were confirmed: TSPO was upregulated in the SDH on day 14 after tumor cell incubation. The single i.t. administration of MZL dose-dependently inhibits thermal hyperalgesia in BCP rats, indicated that spinal astrocytic upregulation of TSPO induced by tumor cell incubation was involed in the analgesic effect of MZL.

Ropi, one of the newest and safest LAs, is widely used for local anesthesia in clinic. However, its relative neurotoxicity limits the clinical use. Accumulative evidence indicates that Ropi is considered as an effective drug in many kinds of pain models, such as CFA-induced chronic inflammatory pain and neuropathic pain $(14,29)$. The analgesic effect of Ropi is contributed to inhibition of the overexpression of spinal c-Fos and GFAP in neuropathic pain that is consistent with previous electrophysiological results. In our study, Ropi dose-dependently attenuated thermal hyperalgesia in the BCP model, together with consequent observations on suppressing spinal neurons and neuroglia activation with the downregulation of spinal pERK.

In our study, the potential synergistically analgesic mechanisms of MZL and Ropi might be as follows. First, many researchers have confirmed that the TSPO upregulation was an endogenous protective response during the process of inflammatory stimulus and/or nerve injury, and MZL binds TSPO to reinforce this 'protective shield' and promotes its analgesic effect (17). TSPO mainly locates on the outer membrane of mitochondria which are the source of reactive oxygen species (ROS), and MZL suppresses the production of ROS and attenuates ROS-induced nerve injury (30). Furthermore, dimethylthiourea, one of ROS scavengers, has the ability to inhibit STAT3 activation induced by focal cerebral ischemiareperfusion injury in rats (31). Hence, it is possible that MZL inhibit spinal pSTAT3 expression through suppression of ROS production via spinal activation of TSPO. A study of rat glial cell immune inflammation showed that MZL inhibited the release of IL- 6 from rat C6 glioma cells by inhibiting astrocytic pSTAT3 activation in TSPO dose-dependently, while increased level of IL-6 and activation of STAT3 have been considered as trigger for initiation and maintaining of pain $(20,32)$. We also found that MZL could obviously inhibit pSTAT3 activation.

Second, spinal pERK activation plays a crucially role in the maintenance of many kinds of pain, such as nerve injury and inflammation, and participates in regulating astrocytic activation and proliferation $(33,34)$. In BCP model, spinal pERK activation was mainly expressed in neurons, but not in microglia and astrocytes in the early phase. However, in the late phase, spinal pERK expression was mainly located in astrocytes (4). In our study, we found that Ropi could downregulate spinal pERK expression with significant inhibition of neurons and neuroglia cells activation.

Third, based on the above evidence, we suggest that synergistical analgesia effect of MZL and Ropi depended on enhancing independent pathways. We showed that either MZL or Ropi could inhibit spinal pSTAT3 and pERK expression with significant inhibition of neurons and neuroglia cell activation in BCP rats. However, MZL rather than Ropi showed selective inhibitory effect on spinal pSTAT3 expression. On the contrary, Ropi predominantly suppressed spinal pERK expression compared with MZL. In addition, the combination of MZL and Ropi produced stronger and longer analgesic effect with lower dose, and showed stronger inhibitory effect on neurons and neuroglia activation.

In light of the available data above, a conservative way to explain this synergistic effect is that Ropi blocks fast voltagegated sodium channels and further inhibits the spinal pERK expression, then MZL binds to TSPO in the outer membrane of mitochondria and the consequent production of ROS mediated by inhibition of spinal pSTAT3 expression. However, there is a gap between animal research and clinical application, the effective doses of drug that works well in animals is not sure in humans. In addition, the safety doses of drug should be confirmed with considered judgments in different groups.

In conclusion, the major finding of our study shows that MZL and Ropi act synergistically to inhibit BCP in rats. Further study is needed to make sure the safe and effective doses of i.t. MZL and Ropi in humans. We propose that the combination of MZL and Ropi might be a novel strategy for the treatment of BCP.

\section{Acknowledgements}

This study was supported by grants from the National Natural Science Foundation of China (nos. 81371112 and 81171050) and Program for Shaanxi Province Key Research Team of Science and Technology Innovation (2012KCT-14).

\section{References}

1. Kane CM, Hoskin P and Bennett MI: Cancer induced bone pain. BMJ 350 (jan29 7): h315, 2015.

2. Schug SA and Chandrasena C: Pain management of the cancer patient. Expert Opin Pharmacother 16: 5-15, 2015.

3. Guan XH, Fu QC, Shi D, Bu HL, Song ZP, Xiong BR, Shu B, Xiang HB, Xu B, Manyande A, et al: Activation of spinal chemokine receptor CXCR3 mediates bone cancer pain through an Akt-ERK crosstalk pathway in rats. Exp Neurol 263: 39-49, 2015.

4. Wang LN, Yao M, Yang JP, Peng J, Peng Y, Li CF, Zhang YB, Ji FH, Cheng H, Xu QN, et al: Cancer-induced bone pain sequentially activates the ERK/MAPK pathway in different cell types in the rat spinal cord. Mol Pain 7: 48, 2011.

5. Liu S, Mi WL, Li Q, Zhang MT, Han P, Hu S, Mao-Ying QL and Wang YQ: Spinal IL-33/ST2 signaling contributes to neuropathic pain via neuronal CaMKII-CREB and astroglial JAK2-STAT3 cascades in mice. Anesthesiology 123: 1154-1169, 2015.

6. Wang ZF, Li Q, Liu SB, Mi WL, Hu S, Zhao J, Tian Y, Mao-Ying QL, Jiang JW, Ma HJ, et al: Aspirin-triggered Lipoxin A4 attenuates mechanical allodynia in association with inhibiting spinal JAK2/STAT3 signaling in neuropathic pain in rats. Neuroscience 273: 65-78, 2014. 
7. Olkkola KT and Ahonen J: Midazolam and other benzodiazepines. Handb Exp Pharmacol 182: 335-360, 2008.

8. Reddy SD and Reddy DS: Midazolam as an anticonvulsant antidote for organophosphate intoxication - A pharmacotherapeutic appraisal. Epilepsia 56: 813-821, 2015.

9. Yilmaz E, Hough KA, Gebhart GF, Williams BA and Gold MS: Mechanisms underlying midazolam-induced peripheral nerve block and neurotoxicity. Reg Anesth Pain Med 39: 525-533 2014.

10. Leone S, Di Cianni S, Casati A and Fanelli G: Pharmacology, toxicology, and clinical use of new long acting local anesthetics, ropivacaine and levobupivacaine. Acta bio-medica. Atenei Parmensis 79: 92-105, 2008.

11. Zink W and Graf BM: The toxicity of local anesthetics: The place of ropivacaine and levobupivacaine. Curr Opin Anaesthesiol 21: 645-650, 2008

12. Sun ZH, Xu XP, Song ZB, Zhang Z, Wang N and Guo QL: Repeated intrathecal administration of ropivacaine causes neurotoxicity in rats. Anaesth Intensive Care 40: 825-831, 2012.

13. Li TF, Fan H and Wang YX: Epidural sustained release ropivacaine prolongs anti-allodynia and anti-hyperalgesia in developing and established neuropathic pain. PLoS One 10: e0117321, 2015

14. Wu HH, Yin JB, Zhang T, Cui YY, Dong YL, Chen GZ and Wang W: Inhibiting spinal neuron-astrocytic activation correlates with synergistic analgesia of dexmedetomidine and ropivacaine. PLoS One 9: e92374, 2014.

15. Wang X, Xu S, Qin X, Li X, Feng SW, Liu Y, Wang W, Guo X, Shen R, Shen X, et al: Comparison between the use of ropivacaine alone and ropivacaine with sufentanil in epidural labor analgesia. Medicine (Baltimore) 94: e1882, 2015.

16. Guo S, Li B, Gao C and Tian Y: Epidural analgesia with bupivacaine and fentanyl versus ropivacaine and fentanyl for pain relief in labor: A meta-analysis. Medicine (Baltimore) 94: e880, 2015.

17. Liu X, Liu H, Xu S, Tang Z, Xia W, Cheng Z, Li W and Jin Y Spinal translocator protein alleviates chronic neuropathic pain behavior and modulates spinal astrocyte-neuronal function in rats with L5 spinal nerve ligation model. Pain 157: 103-116, 2016.

18. Wang DS, Tian Z, Guo YY, Guo HL, Kang WB, Li S, Den YT, Li XB, Feng B, Feng D, et al: Anxiolytic-like effects of translocator protein (TSPO) ligand ZBD-2 in an animal model of chronic pain. Mol Pain 11: 16, 2015.

19. Miao YL, Guo WZ, Shi WZ, Fang WW, Liu Y, Liu J, Li BW, $\mathrm{Wu} \mathrm{W}$ and Li YF: Midazolam ameliorates the behavior deficits of a rat posttraumatic stress disorder model through dual $18 \mathrm{kDa}$ translocator protein and central benzodiazepine receptor and neurosteroidogenesis. PLoS One 9: e101450, 2014

20. Tanabe K, Kozawa $\mathrm{O}$ and Iida $\mathrm{H}$ : Midazolam suppresses interleukin-1 $\beta$-induced interleukin- 6 release from rat glial cells. J Neuroinflammation 8: 68, 2011.
21. Asiedu M, Ossipov MH, Kaila K and Price TJ: Acetazolamide and midazolam act synergistically to inhibit neuropathic pain. Pain 148: 302-308, 2010.

22. Shih A, Miletic V, Miletic G and Smith LJ: Midazolam administration reverses thermal hyperalgesia and prevents gamma-aminobutyric acid transporter loss in a rodent model of neuropathic pain. Anesth Analg 106: 1296-1302, 2008.

23. Yang Y, Li H, Li TT, Luo H, Gu XY, Lü N, Ji RR and Zhang YQ: Delayed activation of spinal microglia contributes to the maintenance of bone cancer pain in female Wistar rats via $\mathrm{P} 2 \mathrm{X} 7$ receptor and IL-18. J Neurosci 35: 7950-7963, 2015.

24. Tallarida RJ: Drug synergism: its detection and applications. J Pharmacol Exp Ther 298: 865-872, 2001.

25. Li F, Liu J, Liu N, Kuhn LA, Garavito RM and Ferguson-Miller S Translocator protein $18 \mathrm{kDa}$ (TSPO): An old protein with new functions? Biochemistry 55: 2821-2831, 2016.

26. Campanella M and Turkheimer FE: TSPO: Functions and applications of a mitochondrial stress response pathway. Biochem Soc Trans 43: 593-594, 2015.

27. Lee JW, Kim LE, Shim HJ, Kim EK, Hwang WC, Min S and Yu SW: A translocator protein $18 \mathrm{kDa}$ ligand, Ro5-4864, inhibits ATP-induced NLRP3 inflammasome activation. Biochem Biophys Res Commun 474: 587-593, 2016.

28. Wei XH, Wei X, Chen FY, Zang Y, Xin WJ, Pang RP, Chen Y, Wang J, Li YY, Shen KF, et al: The upregulation of translocator protein $(18 \mathrm{kDa})$ promotes recovery from neuropathic pain in rats. J Neurosci 33: 1540-1551, 2013.

29. Toda S, Sakai A, Ikeda Y, Sakamoto A and Suzuki H: A local anesthetic, ropivacaine, suppresses activated microglia via a nerve growth factor-dependent mechanism and astrocytes via a nerve growth factor-independent mechanism in neuropathic pain. Mol Pain 7: 2, 2011.

30. Gatliff J and Campanella M: TSPO: Kaleidoscopic 18-kDa amid biochemical pharmacology, control and targeting of mitochondria. Biochem J 473: 107-121, 2016.

31. Batarseh A, Li J and Papadopoulos V: Protein kinase C epsilon regulation of translocator protein $(18 \mathrm{kDa})$ Tspo gene expression is mediated through a MAPK pathway targeting STAT3 and c-Jun transcription factors. Biochemistry 49: 4766-4778, 2010.

32. Liu L, You Q, Tu Y, Tu Y, Li Q, Zheng L, Li X, Gu J and Wang G: Midazolam inhibits the apoptosis of astrocytes induced by oxygen glucose deprivation via targeting JAK2-STAT3 signaling pathway. Cell Physiol Biochem 35: 126-136, 2015.

33. Hu XD, Liu YN, Zhang ZY, Ma ZA, Suo ZW and Yang X: Spinophilin-targeted protein phosphatase-1 alleviated inflammatory pain by negative control of MEK/ERK signaling in spinal cord dorsal horn of rats. J Neurosci 35: 13989-14001, 2015.

34. Han P, Liu S, Zhang M, Zhao J, Wang Y, Wu G and Mi W: Inhibition of spinal interlukin-33/ST2 signaling and downstream ERK and JNK pathways in electroacupuncture analgesia in formalin mice. PLoS One 10: e0129576, 2015. 